\title{
Akuter emotionaler Stress als Auslöser für eine Augeninnendruckerhöhung bei Glaukom
}

\author{
Kevin Gillmann ${ }^{a}$ Kirsten Hoskens ${ }^{a} \quad$ Kaweh Mansouria, b \\ aGlaucoma Research Center, Montchoisi, Swiss Visio Network, Lausanne, Schweiz; \\ ${ }^{b}$ Department of Ophthalmology, University of Colorado School of Medicine, Denver, CO, USA
}

\section{Schlüsselwörter}

Glaukom · IOD · Stress · Angst · mentale Gesundheit · Risikofaktor

\begin{abstract}
Zusammenfassung
Hintergrund: Die stressinduzierte Aktivierung des sympathischen Nervensystems löst eine Kaskade metabolischer Reaktionen aus. Emotionaler Stress ist eine spezielle Form von Stress, bei der der Stressor eine psychische Reaktion auf eine Situation ist, die subjektiv als traumatisch empfunden wird. Stresshormone können vielfältige Wirkungen auf den Körper haben; allerdings ist weiterhin unklar, ob und wie sie sich auf die Physiologie der Augen auswirken. Die vorliegende Arbeit berichtet über einen Fall von schwerer okulärer Hypertension, bei dem emotionaler Stress als einzige Ursache ermittelt wurde, und untersucht mögliche aggravierende Faktoren.
\end{abstract}

Fallbericht: Bei der Patientin handelte es sich um eine 78-jährige Frau vom Persönlichkeitstyp A mit anamnestisch bekanntem pseudoexfoliativen Glaukom, die sich mit einem akuten asymmetrischen Anstieg des Augeninnendrucks (IOD) unmittelbar nach einem familiären Zerwürfnis vorstellte. Zuvor war ihr IOD nach einer tiefen Sklerektomie im rechten Auge und einem Ex-PRESS-Shunt im linken Auge stabil gewesen. Die restliche körperliche Untersuchung war vollständig unauffällig, und die optische Kohärenztomographie des vorderen Augensegments zeigte ein durchgängiges diffuses Sickerkissen. Innerhalb von 24 Stunden war parallel zum Rückgang des Stresslevels eine Beinahe-Normalisierung des IODs zu beobachten.

Schlussfolgerungen: Der vorliegende Fallbericht spricht dafür, dass akuter emotionaler Stress den IOD bei Patienten mit Glaukom stark beeinflussen kann. Dies könnte bei der Versorgung von Glaukompatienten von Bedeutung sein und deutet darauf hin, dass in Glaukomstudien der Persönlichkeitstyp sowie der emotionale und soziale Kontext als weitere Faktoren berücksichtigt werden müssen. Diese Beobachtungen basieren auf einem Einzelfallbericht und müssten in einer größeren Untersuchung bestätigt werden.

(c) The Author(s). 2019

\section{Hintergrund}

Die genauen pathophysiologischen und biologischen Mechanismen, die den glaukomatösen Veränderungen zugrunde liegen, sind noch nicht vollständig geklärt [1]. Nachgewiesenermaßen spielt jedoch der Augeninnendruck (Intraokulardruck, IOD) eine zentrale Rolle bei den Krankheitsprozessen und ein erhöhter IOD ist einer der Hauptrisikofaktoren für die Glaukomprogression [2, 3]. Umgekehrt wurde gezeigt, dass das Erzielen niedriger IODWerte bei Glaukom das Fortschreiten der Krankheit verlangsamt und die langfristige Visusprognose verbessert [4-7]. information@karger.com

www.karger.com/kop

(c) 2020 S. Karger GmbH, Freiburg

Karger ${ }^{\prime \prime}=$
Gemäß medizinischer Definition handelt es sich bei Stress um eine Störung der Homöostase eines Organismus. In Hinblick auf die Multiorgan-Ebene ist seit langem bekannt, dass Hormone, insbesondere Stresshormone, im menschlichen ebenso wie im tierischen Modell zahlreiche unterschiedliche Effekte auf den Organismus haben können [8-11]. Die stressinduzierte Aktivierung des sympathischen Nervensystems löst eine Kaskade von metabolischen Reaktionen aus, die als Stressreaktion bezeichnet werden. Diese Reaktion wird vor allem durch die HypothalamusHypophysen-Nebennieren-Achse vermittelt und beeinflusst die physiologischen Konzentrationen der zirkulierenden Hormone, 
darunter auch Cortisol [12]. Eine Veränderung der Hormonkonzentrationen kann systemische Erkrankungen wie Hypertonie oder Atherosklerose zur Folge haben [13]. Emotionaler Stress ist eine spezielle Form von Stress, bei der der Stressor eine psychische Reaktion auf eine Situation ist, die subjektiv als traumatisch empfunden wird. Seine subjektive Natur und die ethischen Fragen, die damit verbunden sind, wenn menschliche Probanden starkem emotionalem Stress ausgesetzt werden, erschweren die Untersuchung dieser Form von Stress in einem kontrollierten Setting. Es liegen jedoch umfangreiche Berichte darüber vor, wie emotional belastende Situationen die kardiovaskuläre Homöostase beeinflussen [14] und für akute pathologische Veränderungen verantwortlich sein können, wie beispielsewise beim Tako-Tsubo-Syndrom [15]. Trotz dieser Evidenzlage ist noch unklar, ob und wie die metabolischen Veränderungen die okuläre Physiologie beeinflussen können. Etliche kleinere Studien sprechen für einen leichten, jedoch statistisch signifikanten IOD-steigernden Effekt von Stress [16]; umgekehrt finden sich in der Literatur anekdotische Berichte, nach denen Entspannung möglicherweise einen gegenteiligen Effekt hat [17].

In der vorliegenden Arbeit berichten wir über einen Fall, bei dem akuter emotionaler Stress als einziger Auslöser für einen starken IOD-Anstieg ermittelt wurde. Darüber hinaus werden die Implikationen dieses Falles für die zukünftige Erforschung und Behandlung des Glaukoms vor dem Hintergrund des aktuellen Wissensstands erörtert.

\section{Fallbericht}

Eine 78-jährige Frau hispanischer Abstammung mit pseudoexfoliativem Glaukom (PEXG) und exsudativer altersbedingter Makuladegeneration (AMD) stellte sich zur Routinekontrolle mit einem akuten IOD-Anstieg im linken Auge in einer großen ophthalmologischen Spezialklinik vor. An Voroperationen war eine 12 Jahre zuvor erfolgte kombinierte Ex-PRESS-Glaukom-Shuntund Kataraktoperation am linken Auge bekannt. Darüber hinaus war 11 Jahre vor der aktuellen Vorstellung eine kombinierte tiefe Sklerektomie und Kataraktoperation am rechten Auge durchgeführt worden, und 2 Jahre nach der Operation eine Goniopunktion mittels YAG-Laser. Unter einer topischen Therapie mit Latanoprost (Xalatan, Pfizer PFE Switzerland GmbH, Schweiz) und Timolol 0,1\% (Timogel, Théa Pharma SA, Schweiz) lag ihr IOD seitdem relativ stabil zwischen 14 und $19 \mathrm{mmHg}$ im rechten Auge und zwischen 16 und $21 \mathrm{mmHg}$ im linken Auge. Die AMD war nach einer Serie von 3 intravitrealen Injektionen von Ranibizumab im linken Auge (Lucentis, Novartis Pharma, Schweiz), die 4 Monate zuvor erfolgt waren, stabil geblieben.

Bei ihrer Vorstellung in der Klinik war die Patientin asymptomatisch; sie hatte keine Schmerzen oder Beschwerden und die bestkorrigierte Sehschärfe betrug 10/10 im rechten Auge und 7/10 im linken Auge. Bei der Untersuchung mittels Goldmann-Tonometer lag der IOD im rechten Auge bei $18 \mathrm{mmHg}$ und im linken Auge bei $48 \mathrm{mmHg}$ und die Hornhautdicke betrug $552 \mu \mathrm{m}$ und $555 \mu \mathrm{m}$ im rechten bzw. linken Auge.

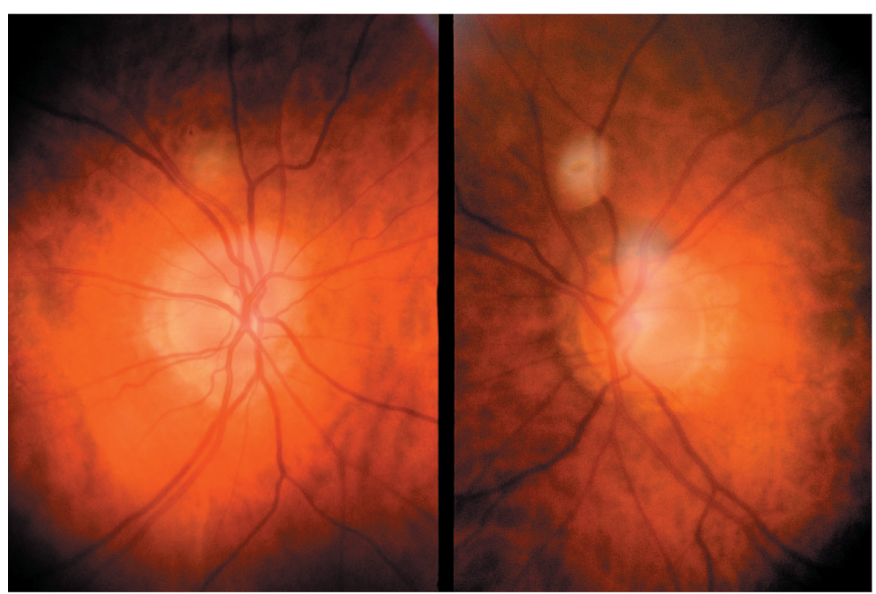

Abb. 1. Aufnahmen der Sehnervenpapille mit asymmetrischen Papillenexkavationen. Der linke Sehnervenkopf (rechtes Bild) weist eine CupDisc-Ratio von 0,7 auf.

Die Spaltlampenuntersuchung ergab ruhige Vorderkammern mit regelrecht liegenden Intraokularlinsen, gut dimensionierte diffuse Sickerkissen in beiden Augen und 2 In-situ-Skleranähte im linken Sickerkissen. Die gonioskopische Untersuchung war unauffällig und zeigte offene Winkel in beiden Augen, mit einer offenen Trabekula-Descemet-Membran im rechten Auge und einem Ex-PRESS Shunt in situ im linken Auge; dessen Lage und Durchgängigkeit wurde mittels optischer Kohärenztomographie (OCT) des vorderen Augensegments (Spectralis OCT, Heidelberg Engineering AG) bestätigt. Die OCT bestätigte außerdem das funktionelle und diffuse Erscheinungsbild der Sickerkissen. Die Fundusuntersuchung, Gesichtsfeldbestimmung und OCTBildgebung zeigten stabile Ergebnisse mit ausgedehnte Drusen in beiden Augen, außerdem kein Rezidiv des Makulaödems, normale Netzhautgefäße und eine Cup-Disc-Ratio von 0,6 im rechten und 0,7 im linken Auge bei einem Papillendurchmesser von 1,4 mm (Abb. 1-3).

Laut anamnestischen Angaben der Patientin hielt sie die Therapie ein und es war kein körperlich traumatisches Ereignis in der jüngeren Vergangenheit aufgetreten. Die Patientin berichtete jedoch von sich aus, dass sie eine Phase schweren emotionalen Stresses durchmache, da es kürzlich zu einem Familienzerwürfnis gekommen sei und sie unmittelbar vor dem Termin eine emotionale Auseinandersetzung gehabt habe. Ihre Persönlichkeit wurde von den Ärzten subjektiv als Typ A eingestuft.

Es wurde eine Kombinationstherapie mit topischem Timolol und Dorzolamid (Cosopt, Santen, Japan) und Brimonidin (Alphagan, Allergan, Dublin, Irland) zweimal täglich im linken Auge und einer täglichen Dosis von 500 mg Acetazolamid (Diamox, Vifor Pharma, Schweiz) oral eingeleitet, um den IOD rasch zu normalisieren und die Nervenfasern zu erhalten.

Am nächsten Tag hatte sich der IOD im rechten und linken Auge auf $10 \mathrm{mmHg}$ bzw. $16 \mathrm{mmHg}$ normalisiert. Die systemischen Medikamente wurden reduziert und abgesetzt, und der IOD ging mit aufeinander folgenden Messwerten zwischen 12 und $16 \mathrm{mmHg}$ im rechten Auge und 18 bis $23 \mathrm{mmHg}$ im linken Auge auf Werte
42

Kompass Ophthalmol 2020;6:41-47 DOI: $10.1159 / 000505946$ 

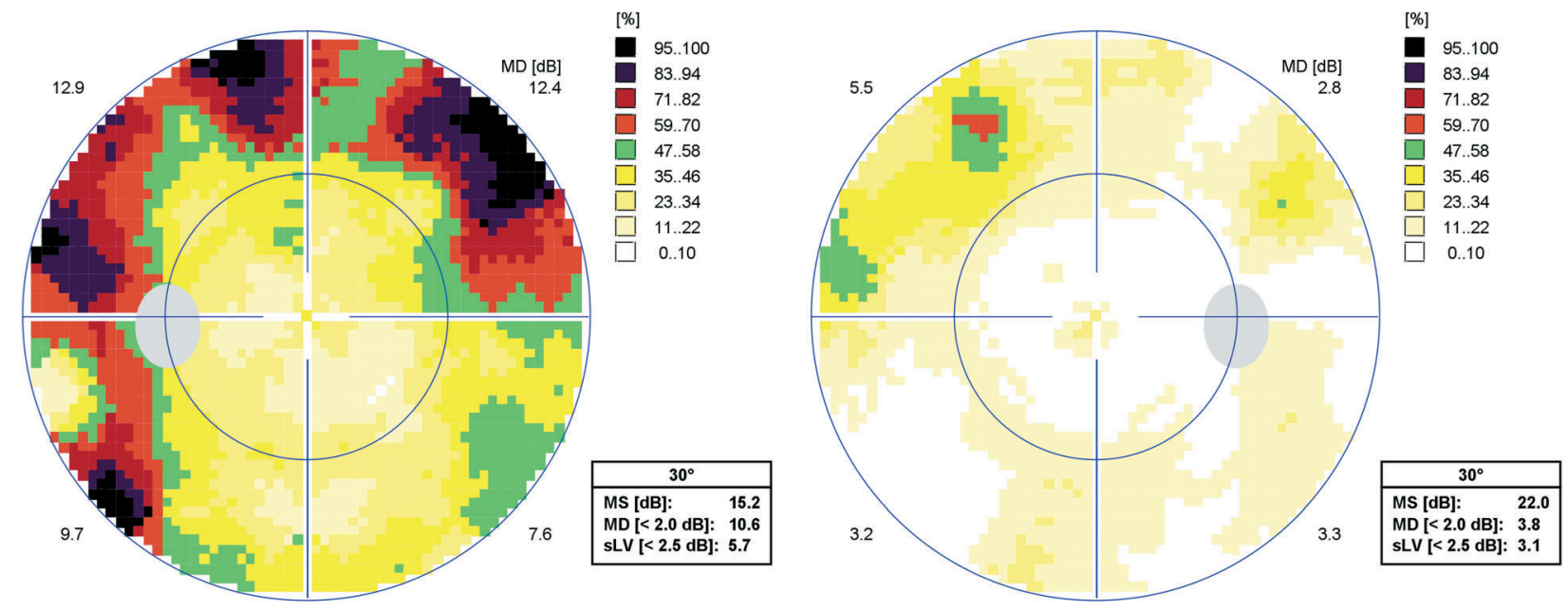

Abb. 2. $30^{\circ}$-Oktopus-Gesichtsfelduntersuchung mit deutlichen superioren und inferioren Bogenskotomen im linken Auge (linkes Bild) mit einer mittleren Abweichung von 10,6 dB, und einem leichten superioren Bogenskotom im rechten Auge (rechtes Bild) mit einer mittleren Abweichung von 3,8 dB. (MS: Mittlere Sensitivität; sLV: Square root of lost variance).
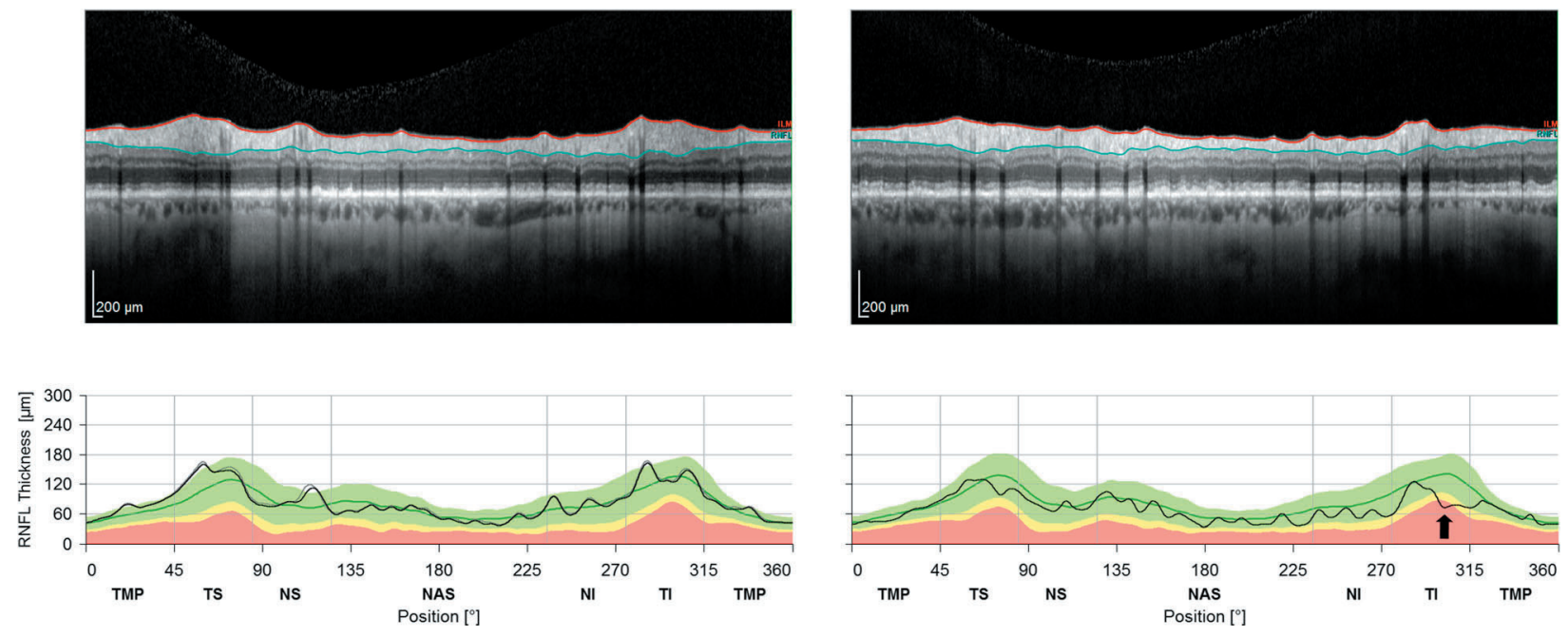

Abb. 3. OCT-Untersuchung der retinalen Nervenfaserschicht (RNFL), die eine leichte generalisierte Ausdünnung der retinalen Nervenfaserschichten zeigt, bei gleichzeitigem Vorliegen eines fokalen inferotemporalen Defekts (schwarzer Pfeil) im linken Auge (rechtes Bild).

nahe den Ausgangswerten zurück (siehe Abb. 1). Unterdessen berichtete die Patientin, dass ihr Stresslevel trotz anhaltender Angstgefühle und einer schwierigen familiären Situation subjektiv leicht abgenommen habe.

Zwei Monate später war der IOD in beiden Augen unter topischem Timolol und Dorzolamid im linken Auge weiterhin stabil (Abb. 4). Um Schwankungen zwischen den Messungen und über den Tag auftretende Schwankungen auszuschließen, erfolgte eine 24-Stunden-Messung der IOD-Schwankungen mittels Triggerfish-Kontaktlinsensensor (Sensimed SA, Lausanne, Schweiz).
Diese zeigte relativ stabile Druckwerte über den Tag und in der Nacht, mit minimalen Veränderungen nach dem Einbringen der topischen Therapie (Abb. 5). Zur weiteren Verbesserung der IODKontrolle im linken Auge wurde ein Needling des hinteren Augensegments in Kombination mit einer Lösung der Naht durchgeführt, doch trotz einer leichten initialen Verbesserung stabilisierte sich der IOD nach 2 Monaten bei $22 \mathrm{mmHg}$. Es erfolgte eine XEN-unterstützte Baerveldt-Operation, mit der ein IOD ohne medikamentöse Therapie nach einem Monat von $16 \mathrm{mmHg}$ erreicht wurde [18]. 

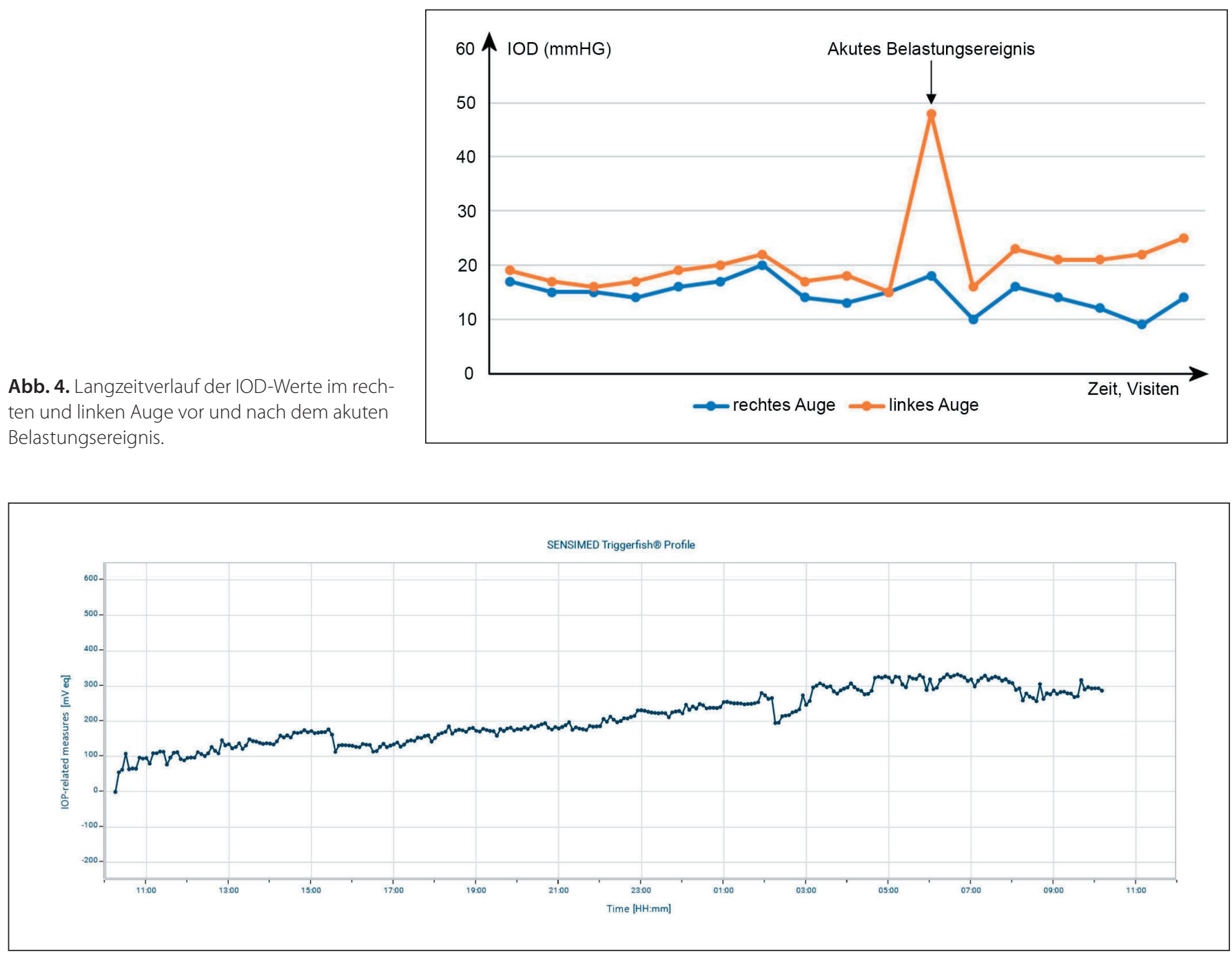

Abb. 5. 24-Stunden-Kurve der IOD-Schwankungen im linken Auge, gemessen mittels Kontaktlinsensensor. Triggerfish-Sensoren messen Formveränderungen der Hornhaut (in mV), die Tendenzen der Augeninnendruckschwankungen widerspiegeln. Die oben dargestellte Kurve zeigt eine relative Stabilität über die gesamte Aufzeichnungsdauer.

\section{Diskussion und Schlussfolgerungen}

Dieser Fallbericht zeigt, dass starker emotionaler Stress akute Auswirkungen auf die IOD-Kontrolle bei Patienten mit PEXG haben kann. Dies wird vor allem durch das Fehlen einer nachgewiesenen anatomischen oder physiologischen Abflussbehinderung bei gleichzeitig funktionsfähigen großen Sickerkissen gestützt. Allerdings ist das PEXG für seine Instabilität und Aggressivität bekannt, und die anhaltende Dekompensation, die durch nicht identifizierte Faktoren aufrechterhalten wird, spricht dafür, dass gemischte Mechanismen beteiligt sind. Die Tatsache, dass die IOD-Werte vor dem belastenden Ereignis relativ stabil waren und die okuläre hypertensive Krise mit zunehmender Besserung der emotionalen Situation der Patientin fast vollständig abklang, sind jedoch weitere Indikatoren dafür, dass emotionaler Stress zu der akuten Krise zumindest beigetragen hat.

Im vorliegenden Fall fällt die Reaktion eindeutig asymmetrisch aus, mit einem deutlich stärkeren IOD-Anstieg im linken Auge als im rechten Auge. Zwar liegen in der Literatur etliche Berichte über IOD-Abweichungen zwischen den Augen von Patienten mit bilateraler Glaukomoperation vor [19, 20]; dennoch war diese Beobachtung eher unerwartet. Sie könnte auf mehrere Unterschiede zwischen den beiden Augen zurückzuführen sein: Erstens postoperative strukturelle Unterschiede. Am rechten Auge war eine nicht-penetrierende tiefe Sklerektomie mit anschließender Goniopunktion mittels Nd:YAG-Laser erfolgt, durch die eine direkte Verbindung zwischen Vorderkammer und subkonjunktivalem Raum geschaffen wurde, wohingegen am linken Auge eine ExPRESS-Shunt-Implantation durchgeführt worden war. Bei diesem Eingriff wird eine ähnliche Technik verwendet wie bei der perforierenden Trabekulektomie, nur dass nach Anhebung des Sklerallappens ein Metallshunt anstelle eines Skleralochs verwendet wird, um Zugang zur Vorderkammer zu erhalten [21]. Zwar ist der Ex-PRESS Shunt mit einem höheren intrinsischen Widerstand verbunden als die Trabekulektomie, doch weist er unter physiologischen Bedingungen einen äußerst geringen Abflusswi- 
derstand (0,09 mmHg Widerstand) auf, und erfordert ebenfalls eine Skleralappennaht, damit es unmittelbar postoperativ nicht zu einer Hypotonie kommt [22]. Die Spannung im Skleralappen bewirkt einen größeren Abflusswiderstand als durch das offene Filtrationsmodell einer tiefen Sklerektomie nach Goniopunktion erzeugt wird, was die Unterschiede zwischen den beiden Augen erklären könnte. Der stressbedingte Anstieg des IODs in einem Auge mit Filtrationsoperation, bei dem somit das episklerale Venensystem umgangen wird, zeigt, dass Stress sich nicht nur auf den Abflusswiderstand auswirkt, sondern auch auf die Geschwindigkeit der Kammerwasserproduktion, wie dies bereits in einem Tiermodell von Niederer et al. [23] festgestellt wurde. Zum Zweiten könnte auch die kürzlich erfolgte einseitige Exposition gegenüber Ranibizumab zu dem beobachteten Unterschied im IOD zwischen beiden Augen beigetragen haben. In einer kürzlich veröffentlichten Studie wiesen Foss et al. [24] nach, dass Anti-VEGFTherapien nach jeder intravitrealen Injektion einen dauerhaften und kumulativen IOD-Anstieg verursachten, was zur Folge hatte, dass der Unterschied im IOD zwischen dem behandelten und dem unbehandelten Auge kontinuierlich zunahm. Der beschriebene Effekt war zwar statistisch signifikant, jedoch relativ gering und somit vermutlich nicht allein für die in unserem Fall beobachtete Abweichung verantwortlich. Anderen Studien zufolge gingen Anti-VEGF-Wirkstoffe jedoch mit einer verminderten Bildung von Prostaglandin-I 2 (PGI2) [25] einher, ein Prostaglandin-Typ, der im Tiermodell einige der Cortisol-Wirkungen unterdrückte [26]. Daher ist anzunehmen, dass Anti-VEGF-Therapien über die verringerte PGI2-Konzentration zu einer ungehinderten Wirkung von Cortisol führen können, wodurch dessen Wirkung lokal verstärkt wird und die Auswirkungen von Stress potenziell zunehmen. Und schließlich könnte auch die Schwere der Erkrankung zu den IOD-Unterschieden zwischen beiden Augen beitragen. Gottanka et al. zeigten in ihrer Studie, dass der IOD-Höchstwert in Augen mit pseusoexfoliativem Glaukom direkt mit dem Ausmaß der axonalen Schädigung am Sehnerv und der Menge an pseudoexfoliativem (PEX) Material im Schlemm-Kanal korrelierte [27]. Im vorliegenden Fall wurde im linken Auge, das einen höheren klinischen PEXG-Schweregrad aufwies, ein höherer IOD festgestellt. Dies hängt vermutlich mit dem vermehrten PEX-Material im Schlemm-Kanal zusammen, was ein weiterer Faktor für einen höheren IOD in diesem Auge sein könnte.

Verschiedenen Studien zufolge können hormonelle Schwankungen und emotionale Reaktionen den IOD im menschlichen Modell beeinflussen, wie dies von Brody et al. gezeigt wurde. Sie beobachteten einen IOD-Anstieg um 1,3 mmHg, nachdem die Probanden aufgefordert worden waren, komplexe Kopfrechenaufgaben zu lösen [28] und Mansouri et al. berichteten über einen deutlichen IOD-Rückgang während sexueller Aktivität und beim Orgasmus [29]. Die Auswirkungen von emotionalem Stress auf den IOD sind jedoch weniger gut dokumentiert und die Studienergebnisse fallen widersprüchlich aus.

Méndez-Ulrich et al. stellten 2018 fest, dass der IOD in einer Gruppe von Probanden, die sich selbst als nervös einstuften, durchschnittlich 2,3 mmHg höher war als in der Gruppe mit niedrigem Angstlevel [30]. Die erste Gruppe wies zudem eine höhere Herzfrequenz auf als die zweite Gruppe, aber es zeigte sich kein signifikanter Unterschied in den Blutdruckwerten. Eine frühere Studie von Ismail et al. stützte dieses Ergebnis, denn sie beobachteten, dass ein melatoninvermittelter Rückgang der Angst vor der Kataraktoperation mit einer ähnlichen IOD-Abnahme verbunden war. Allerdings fanden sie eine statistisch signifikante Senkung des mittleren arteriellen Blutdrucks, jedoch keine Veränderung der Herzfrequenz [31]. In dieser Studie wurde der IOD $\mathrm{zu}$ verschiedenen Zeitpunkten gemessen, unter anderem $90 \mathrm{Mi}$ nuten vor der Operation (vor Gabe der Prämedikation), beim Eintritt in den Operationssaal sowie postoperativ. Interessanterweise zeigte sich in der Kontrollgruppe, die keine Medikamente erhalten hatte, keine statistisch signifikante Abweichung der IODMesswerte, obwohl zu erwarten wäre, dass sich die Angstzustände beim Eintritt in den Operationssaal und nach Ende der Operation unterscheiden. In einer ähnlichen Studie über die Wirkung von Melatonin auf Stress und den IOD widersprechen Khezri et al. diesen Ergebnissen. Ihren Beobachtungen zufolge verminderte die pharmakologische Angstreduktion die kardiovaskulären Auswirkungen, hatte jedoch keinen signifikanten Einfluss auf den IOD [32].

Die unterschiedlichen Beobachtungen hinsichtlich der Auswirkungen von Angstzuständen sind auf mehrere Faktoren zurückzuführen. Erstens ist emotionaler Stress eine subjektive Erfahrung und es kann schwierig sein vorherzusagen, was von der Mehrheit der Probanden als belastend empfunden wird, wie dies Terracciano et al. in ihrer Studie über die Inzidenz des WeißkittelSyndroms bei verschiedenen Persönlichkeitstypen feststellten, in der sie beobachteten, dass ängstliche Persönlichkeitstypen den klinischen Kontext einer Gesundheitsuntersuchung tendenziell beruhigend finden [33]. Zweitens wurde gezeigt, dass die Persönlichkeitstypen und das Alter einen signifikanten Einfluss auf die IOD-Schwankungen haben können, wobei Typ-A-Persönlichkeitstypen die größte Variabilität aufweisen [34]. In den meisten überprüften Studien wurden diese Merkmale nicht analysiert und sie beinhalteten keine Bereinigung für diese Merkmale. Und schließlich ist bekannt, dass Patienten, die an einem Glaukom leiden, und in noch stärkerem Maße PEXG-Patienten, größere tageszeitliche IOD-Schwankungen aufweisen [35]. In den überprüften Studien zu Melatonin waren glaukomatöse Augen nicht ausdrücklich ein- oder ausgeschlossen, doch könnte der Anteil glaukomatöser Augen die Ergebnisse unter Umständen beeinflussen.

Unser Fall zeigt in diesem Zusammenhang, dass akuter emotionaler Stress eine starke okuläre Reaktion in Form eines ausgeprägten IOD-Anstiegs auslösen kann. Das Ausmaß der Reaktion auf Stressreize wird möglicherweise durch verschiedene Faktoren beeinflusst, darunter die Diagnose Glaukom, die Art des Glaukoms, Voroperationen am Auge, der Persönlichkeitstyp des Patienten und die empfundene Schwere der emotionalen Belastung. Dies könnte bei der Versorgung von Glaukompatienten wichtig sein, insbesondere bei jüngeren Patientenkollektiven, bei denen die Diagnose Glaukom tendenziell mit höheren Angstzuständen 
verbunden ist [36]. Außerdem zeigt es, dass in Glaukomstudien der Persönlichkeitstyp sowie der emotionale und soziale Kontext als weitere Faktoren berücksichtigt werden müssen. Diese Beobachtungen und Annahmen basieren auf einem Einzelfallbericht und müssten in größeren Untersuchung mit einem strukturierten und standardisierten Ansatz bestätigt werden.

\section{Danksagung}

Unterstützt durch die Swiss Glaucoma Research Foundation, Lausanne, Schweiz.

\section{Finanzielle Unterstützung}

Die Autoren haben keine Finanzierung für diese Arbeit erhalten.

\section{Autorenbeiträge}

KG: Datenanalyse, Überprüfung der Literatur und reaktionelle Bearbeitung des Manuskripts. KH: Klinische Behandlung des Patienten, Followup und Datenerhebung. KM: Ursprüngliche Idee, Aufsicht, Korrekturlesung und Expertenhinweise. Alle Autoren haben die finale Fassung des Manuskripts gelesen und freigegeben.

\section{Zustimmung zur Veröffentlichung}

Von der Patientin wurde nach Aufklärung eine schriftliche Einverständniserklärung für die Veröffentlichung der vorliegenden Arbeit und der dazugehörigen Bilder eingeholt. Der Wortlaut der schriftlichen Einverständniserklärung ist von der Redaktion dieser Zeitschrift zur Überprüfung erhältlich.

\section{Interessenkonflikte}

Die Autoren erklären, dass keine Interessenkonflikte bestehen.

\section{Lizenzangabe}

Kevin Gillmann, Kirsten Hoskens, Kaweh Mansouri: Acute emotional stress as a trigger for intraocular pressure elevation in glaucoma. BMC Ophthalmol 2019;19:69 (https://doi.org/10.1186/s12886-019-1075-4), ${ }^{\circ}$ The Author(s). 2019 (Übersetzung, «Abbrevations», «Availability of data materials», "Ethics approval and consent to participate» und «Publisher's Note» gekürzt), lizensiert unter CC BY 4.0 (https://creativecommons.org/licenses/ by/4.0/deed/de).

\section{Literatur}

1 Weinreb RN, Aung T, Medeiros FA: The pathophysiology and treatment of glaucoma: a review. JAMA 2014;311:1901-1911.

-2 Boland MV, Ervin AM, Friedman DS, et al.: Comparative effectiveness of treatments for open-angle glaucoma: a systemic review for the US preventive services task force. Ann Intern Med 2013;158:271-279.

3 Nickells RW, Howell GR, Soto I, et al.: Under pressure: cellular and molecular responses during glaucoma, a common neurodegeneration with axonopathy. Annu Rev Neurosci. 2012;35:153-179.

4 Collaborative Normal-Tension Glaucoma Study Group: The effectiveness of intraocular pressure reduction in the treatment of normaltension glaucoma. Am J Ophthalmol. 1998; 126:498-505.

$\checkmark 5$ Heijl A, Leske MC, Bengtsson B, et al.: Reduction of intraocular pressure and glaucoma progression: results from the early manifest Glaucoma trial. Arch Ophthalmol. 2002;120: $1268-1279$.

6 Investigators TAGIS. The advanced Glaucoma intervention study (AGIS): 7. The relationship between control of intraocular pressure and visual field deterioration.The AGIS Investigators. Am J Ophthalmol 2000;130: 429-440.

7 Musch DC, Gillespie BW, Lichter PR, et al.: Visual field progression in the collaborative initial Glaucoma treatment study the impact of treatment and other baseline factors. Ophthalmol. 2009;116:200-207

$\checkmark 8$ Vors O, Marqueste T, Mascret N.: The Trier social stress test and the Trier social stress test for groups: qualitative investigations. PLoS One. 2018;13:e0195722.

$\checkmark 9$ Yeager MP, Guyre CA, Sites BD, et al.: The stress hormone cortisol enhances interferon- $v$ - mediated Proinflammatory responses of human immune cells. Anesth Analg. 2018;127: 556-563.

10 Naish KR, Laliberte M, MacKillop J, et al.: Systematic review of the effects of acute stress in binge eating disorder. Eur J Neurosci. 2019. 10.1111/ejn.14110.

11 Ensminger DC, Owen DAS, MacLeod KJ, et al.: Maternal stress alters the phenotype of the mother, her eggs, and her offspring in a wild caught lizard. J Anim Ecol 2018;87: 1685-1697.

$\checkmark 12$ Miller DB, O’Callaghan JP: Neuroendocrine aspects of the response to stress. Metabolism. 2002;51(6 Suppl 1):5-10.

13 Ayari H: Respective roles of cortisol, aldosterone and angiotensin II during pathophysiology of atherosclerosis. Ann Biol Clin (Paris) 2013;71:381-388.

14 Meyer FU: Haemodynamic changes under emotional stress following a minor surgical procedure under local anesthesia. Int J Oral Maxillofac Surg 1988;16:688-694.

15 Doue T, Ito K, Yuba T, et al.: A case of “Takotsubo" cardiomyopathy observed with myocardial scintigraphy from the acute phase. Kaku Igaku 2002;39:511-518.

16 Jiménez R, Vera J: Effect of examination stress on intraocular pressure in university students. Appl Ergon 2018;67:252-258.

17 Bertelmann T, Strempel I: Short-term effects of relaxation music on patients suffering from primary open-angle glaucoma. Clin Ophthalmol 2015;9:1981-1988.

18 D’Alessandro E, Guidotti JM, Mansouri K, et al.: XEN-augmented Baerveldt: a new surgical technique for refractory Glaucoma. J Glaucoma. 2017;26:e90-e92.

19 Jung Y, Park HY, Lee NY, et al.: Difference in outcomes between first-operated vs. fellow- operated eyes in patients undergoing bilateral Trabeculectomies. PLoS One. 2015; 10:e0136869.

20 Din NM, Talat L, Isa H, et al.: Difference in glaucoma progression between the first and second eye after consecutive bilateralglaucoma surgery in patients with bilateral uveitic glaucoma. Graefes Arch Clin Exp Ophthalmol 2016;254:2439-2448.

21 Sarkisian SR: The ex-Press mini Glaucoma shunt: technique and experience. Middle East Afr J Ophthalmol 2009;16:134-137.

22 Sheybani A, Reitsamer H, Ahmed I: Fluid dynamics of a novel micro-fistula implant for the surgical treatment of Glaucoma. Invest Ophthalmol Vis Sci 2015;56:4789-4795.

23 Niederer W, Richardson BP, Donatsch P: Hormonal control of aqueous humour production. Exp Eye Res 1975;20:329-340.

24 Foss AJ, Scott LJ, Rogers CA, et al.: Changes in intraocular pressure in study and fellow eyes in the IVAN trial. Br J Ophthalmol 2016;100: 1662-1667.

25 Zarbin MA: Anti-VEGF agents and the risk of Arteriothrombotic events. Asia Pac J Ophthalmol (Phila) 2018;7:63-67.

26 Blajchman MA, Senyi AF, Hirsh J, et al.: Shortening of the bleeding time in rabbits by hydrocortisone caused by inhibition of prostacyclin generation by the vessel wall. J Clin Invest 1979;63:1026-1035.

27 Gottanka J, Flügel-Koch C, Martus P, et al.: Correlation of pseudoexfoliative material and optic nerve damage in pseudoexfoliation syndrome. Invest Ophthalmol Vis Sci 1997;38: 2435-2446.

28 Brody S, Erb C, Veit R, et al.: Intraocular pressure changes: the influence of psychological stress and the Valsalva maneuver. Biol Psychol 1999;51:43-57. 
29 Mansouri K, Medeiros FA, Weinreb RN: Intraocular pressure changes during sexual activity. Acta Ophthalmol. 2013;91:e324-e325.

30 Méndez-Ulrich JL, Sanz A, Feliu-Soler A, et al. Could white coat ocular hypertension affect to the accuracy of the diagnosis of Glaucoma? Relationships between anxiety and intraocular pressure in a simulated clinical setting. Appl Psychophysiol Biofeedback 2018;43:49-56.

31 Ismail SA, Mowafi HA: Melatonin provides anxiolysis, enhances analgesia, decreases intraocular pressure, and promotes better operating conditions during cataract surgery under topical anesthesia. Anesth Analg 2009; 108: 1146-1151.

32 Khezri MB, Merate H: The effects of melatonin on anxiety and pain scores of patients, intraocular pressure, and operating conditions during cataract surgery under topical anesthesia. Indian J Ophthalmol 2013;61:319-324

33 Terracciano A, Scitero A, Strait J, et al.: Are personality traits associated with white coat and masked hypertension? J Hypertens 2014; 32:1987-1992.
34 Bubella RM, Bubella DM, Cillino S: Type a behavior pattern: is it a risk factor for open-angle chronic glaucoma? J Glaucoma 2014;23:199-201.

35 Tojo N, Abe S, Miyakoshi M, et al.: Comparison of intraocular pressure fluctuations before and after $\mathrm{ab}$ internontrabeculectomy in pseudoexfoliation glaucoma patients. Clin Ophtalmol 2017;11:1667-1675.

36 Mabuchi F, Yoshimura K, Kashiwagi K, et al.: Risk factors for anxiety and depression in patients with glaucoma. Br J Ophthalmol 2012; 96:821-825. 